\title{
Marginal Adaptation and Color Stability of Four Provisional Materials
}

\author{
Edward J. Givens, Jr., DDS, ${ }^{1}$ Gisele Neiva, DDS, MS, ${ }^{2}$ Peter Yaman, DDS, MS ${ }^{2}$ \\ \& Joseph B. Dennison, DDS, $\mathrm{MS}^{2}$ \\ 1 United States Navy \\ 2 School of Dentistry, The University of Michigan, Ann Arbor, Ml
}

\section{Keywords}

Marginal fit; color stability; provisional

materials.

\author{
Correspondence \\ Edward Givens Jr., 17 Sherwood Ln, Lady's \\ Island, SC 29907. E-mail: \\ edward.givens@med.navy.mil \\ IADR Oral Poster Discussion, 83rd \\ International Meeting, Honolulu, HI. \\ Funding provided by University of Michigan, \\ Student Research Program and NIH, NIDCR \\ Training Grant \#DE-07101.
}

Accepted August 22, 2006

doi: $10.1111 / j .1532-849 X .2007 .00256 . x$

\begin{abstract}
Purpose: A number of provisional restorative materials exist on the market. This study tested marginal fit and color stability of three provisional restorative materials and a control.

Materials and Methods: Two auto-cure materials, Protemp Garant and Integrity, and one dual-cure material, Luxatemp Solar, were tested against SNAP, a polyethyl methacrylate control. A maxillary right central incisor ivorine tooth was prepared for a full coverage all-ceramic crown, with a $1.5-\mathrm{mm}$ chamfer margin. Four points were engraved at a point $1 \mathrm{~mm}$ below the facial, lingual, mesial, and distal margins, and replicas $(n=40)$ were produced by the manufacturer. Provisional crowns $(n=10 \times$ 4) were fabricated on the individual replicas using a polyethylene coping template. The crowns were trimmed under magnification using an acrylic bur. The distance from the crown margins to a point tangent to the engraved markings was measured under $10 \times$ magnification and recorded. For color stability, 10-mm diameter $\times 2$-mm thick discs $(n=10 \times 4)$ were fabricated and immersed cyclically in tea for 1 week in a Tucillo/Nielson apparatus. Color measurements were recorded for each specimen at baseline and after staining. $\Delta \mathrm{E}$ values were calculated to determine the extent of the color change.

Results: The means of the four marginal discrepancy measurements for each specimen were analyzed using a 1-way ANOVA and Tukey post-hoc comparison. Luxatemp Solar had statistically significant marginal discrepancy $(319 \mu \mathrm{m}, p<0.05)$ when compared to the other provisional materials. A significant color change $(\Delta \mathrm{E}=4.33$, $p<0.05$ ) was found for Protemp Garant.

Conclusion: The dual-cure temporary material (Luxatemp Solar) exhibited significantly more discrepancy at the margin than the auto-cure bis-acryl materials or acrylic control. Protemp Garant exhibited a clinically noticeable change in shade after 1 week in staining solution, whereas the other materials did not exhibit a clinically noticeable change.

Clinical Implications: Provisional crowns fabricated from SNAP, Protemp Garant, and Integrity exhibited similarly low marginal discrepancy. SNAP, Luxatemp Solar, and Integrity did not demonstrate a clinically detectable change in shade after 1 week in a staining solution.
\end{abstract}

Polyethyl- and polymethyl-methacrylates (PEMA and PMMAs) have been a popular choice as provisional materials for temporization of direct and indirect restorative procedures. The more modern auto-cure bis-acryl provisional materials appear to have comparable marginal discrepancy to that of a PEMA material. The bis-acryl provisional materials also have comparable color stability when compared with a PEMA material.

\section{Introduction}

Provisional restorative materials are an important adjunct for a variety of indirect dental procedures, such as inlays, onlays, crowns, bridges, and implant temporization. A provisional helps stabilize prepared teeth during occlusal function and prevents exposure of dental tissues to the harsh conditions found within the oral cavity while the permanent restoration is 
being fabricated. ${ }^{1}$ Provisional materials are particularly important during long-term anterior temporization. They represent an important step in deciding the shade, shape, and contour of the final restoration, especially in complex esthetic reconstructions. $^{2}$

To successfully satisfy the aforementioned requirements, these materials should possess a number of ideal mechanical and physical properties, such as a high flexural strength, increased resistance to wear, high fracture strength, dimensional stability, minimal marginal gap formation, and increased resistance to staining and discoloration. ${ }^{3,4}$

Traditionally, thermoplastic acrylic [PMMA and methyl methacrylate (MMA)] materials have been used as the provisional material of choice and have, to a certain degree, met many of these mechanical and physical requirements. The more modern bis-acryl composite temporization materials, however, have become an increasingly popular choice, due in part to their improved mechanical properties and ease of handling. ${ }^{5-7}$

A variety of techniques exist for fabricating provisional materials. ${ }^{8-12}$ A direct and an indirect technique appear to be the most common. While several studies have used both, there does not seem to be any indication in the literature as to which technique is most accurate, with advantages and disadvantages cited for each one. ${ }^{9-12}$

A limited number of in vitro studies have been conducted to assess the degree of marginal gap formation of both acrylic and bis-acryl resin provisional materials. Koumjian and Holmes, using an indirect technique, tested five acrylic resins, one autocure bis-acryl resin, and one light-cured composite resin. ${ }^{13} \mathrm{Re}$ sults from this study revealed that provisional crowns fabricated from the acrylic resins demonstrated the least amount of marginal discrepancy. Provisional crowns fabricated from the bis-acryl composite had significantly more gap formation than each of the three acrylic resins. In contrast, results from a separate investigation of gap formation revealed a bis-acryl material having statistically similar low marginal discrepancy when compared with two acrylic resin materials. ${ }^{14}$

A number of studies have looked at the color stability of both acrylic and bis-acryl materials under a variety of conditions, such as cyclic immersion through staining solutions, as well as accelerated aging with ultraviolet (UV) light irradiation. ${ }^{15-18}$ Results from these studies suggest that the acrylic resin provisional materials tend to be more resistant to changes in color when subjected to staining through immersion in solution, whereas the bis-acryl composite resins tend to be more resistant to discoloration when exposed to UV light irradiation. These findings are probably related to the mode of discoloration between the two types of staining conditions, wherein cyclic immersion through a solution of staining material occurs through sorption processes, and discoloration from UV light sources occurs through a bulk deterioration process.

The purpose of the present in vitro study was to assess the marginal discrepancy and color stability of two auto-cure and one dual-cure bis-acryl composite temporary materials. It was hypothesized that a PEMA provisional material (control) would exhibit significantly less shrinkage at the margin than the bis-acryl composite materials, and that the PEMA material would be more resistant to changes in color than the bis-acryl materials.
Table 1 Materials used

\begin{tabular}{llll}
\hline Manufacturer & Brand & Material type & Lot \# \\
\hline Parkell, Farmingdale, NY & SNAP & Poly-ethyl methacrylate & 030167 \\
DMG/Zenith, Hamburg, & $\begin{array}{c}\text { Luxatemp } \\
\text { Solar }\end{array}$ & Dual-cure bis-acryl & 515654 \\
$\begin{array}{c}\text { Germany } \\
\text { 3M/ESPE, St. Paul, MN }\end{array}$ & $\begin{array}{c}\text { Protemp } \\
\text { Garant }\end{array}$ & Auto-cure bis-acryl & 148902 \\
Dentsply, Milford, DE & $\begin{array}{l}\text { Integrity } \\
\text { Auto-cure bis-acryl }\end{array}$ & 030402 \\
\hline
\end{tabular}

\section{Materials and methods}

Two auto-cure bis-acryl materials and one dual-cure bis-acryl material were tested against a PEMA control (Table 1). A maxillary right central incisor ivorine tooth (Columbia Dentoform Corp., Long Island City, NY) was prepared for a full coverage all-ceramic restoration with a $1.5-\mathrm{mm}$ chamfer margin. Four points were engraved at a distance of $1 \mathrm{~mm}$ below the facial, lingual, mesial, and distal margins, and replicas were fabricated by the manufacturer (Columbia Dentoform Corp.). Provisional crowns $(n=10 \times 4)$ were fabricated directly on the individual replicas using a template made from a polyethylene coping material. Each crown was fabricated according to manufacturer's specifications. The PEMA powder was saturated with liquid monomer and hand mixed. Material was poured in the template and allowed to become matte finish before being placed over the replica. The bis-acryl material was dispensed from a cartridge through a mixing tip using a dispensing gun and then placed in like manner into the template and onto the replica. A thin layer of petroleum jelly (Vaseline, Unilever PLC, London, England) was applied to each replica, to serve as a releasing agent. Firm hand pressure was applied to the template over the adjacent lateral and left central incisor while the materials were polymerizing. Additionally, the provisionals were slightly elevated and depressed from the abutment replicas during polymerization to simulate the direct technique of fabrication. Upon completion of cure, the crowns were trimmed under magnification $(2.5 \times)$ using an acrylic bur in a slow speed handpiece (Fig 1). No other finishing or polishing procedures were performed on the crown margins.

The specimens were then placed under magnification $(10 \times$, Nikon stereoscopic microscope, Nikon Corp., Tokyo, Japan), and photographed (Nikon camera, Nikon Corp.). The resultant 35-mm slides were scanned onto CD-ROMs (SuperCool Scanner, Nikon Corp.), and a software program, Scion Image (Scion Corporation, Frederick, MD), was used to calculate the distance from the crown margins to a point tangent to the engraved markings (Fig 2). The photograph of the first specimen had a millimeter ruler placed in the field of view to calibrate the computer program and measure the distance of marginal discrepancy. Results from each surface were compiled, and the average of four surfaces was calculated. Finally, an overall average of marginal gap formation was calculated for each test group. These measurements were analyzed using 1-way ANOVA with a Tukey post-hoc multiple comparison, to determine statistical significance.

For color stability, $10-\mathrm{mm}$ diameter $\times 2$-mm thick PEMA and bis-acryl discs $(n=10 \times 4)$ were fabricated using a metal mold 


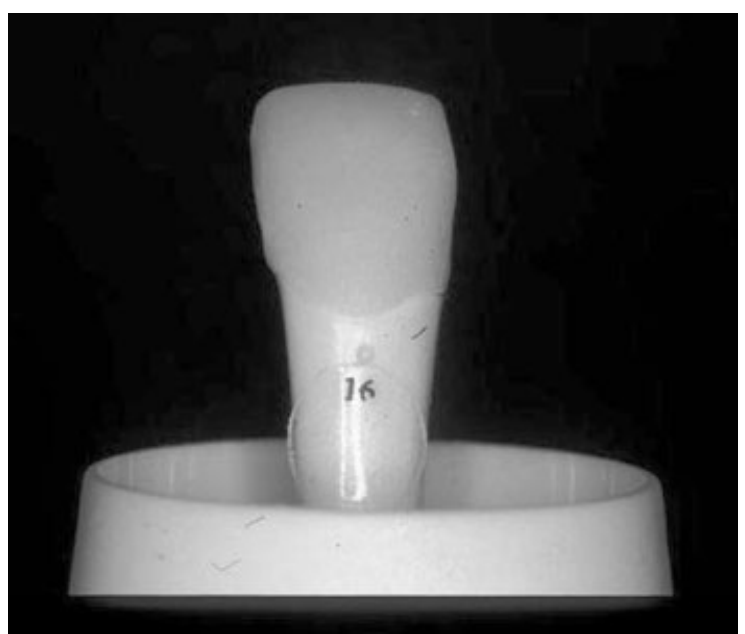

Figure 1 Each provisional crown was fabricated directly over a maxillary right central incisor ivorine tooth. A total of four reference points were created below the facial, lingual, mesial, and distal margins, and were used to calculate marginal discrepancy.

and polished with the Enhance polishing system (Dentsply Caulk, Dentsply International, York, PA). Specimens were fabricated and stored in a light-protected container until ready for testing. Groups of five discs were positioned in a poly(vinyl siloxane) circular stent that was adapted to a Tucillo/Nielson apparatus. The machine rotated the specimens through a high concentration tea solution at a rate of $1 \mathrm{rpm}$. The staining regimen lasted for 1 week, and the solution was constantly monitored and supplemented as needed. Baseline and poststain color readings were obtained for each specimen with the use of a colorimeter (Minolta CR-300 series Colorimeter, Minolta Corp., Ramsey NJ). Color change was then evaluated using the CIE $\mathrm{L}^{*} \mathrm{a}^{*} \mathrm{~b}^{*}$ color system. $\mathrm{L}^{*} \mathrm{a}^{*} \mathrm{~b}^{*}$ values were collected and used to determine $\Delta \mathrm{E}$ values. A $\Delta \mathrm{E}$ value $>3.2$ was used to determine whether a clinically noticeable change in shade occurred. ${ }^{19}$

\section{Results}

The mean gap formation for each group is depicted in Figure 3. SNAP (PEMA control; $177 \mu \mathrm{m}, \mathrm{SD}=63 \mu \mathrm{m}$ ), Protemp Garant $(223 \mu \mathrm{m}, \mathrm{SD}=59 \mu \mathrm{m})$, and Integrity $(218 \mu \mathrm{m}, \mathrm{SD}=65$ $\mu \mathrm{m})$ all exhibited comparable marginal discrepancy. Luxatemp Solar, however, had significantly greater marginal discrepancy (319 $\mu \mathrm{m}, \mathrm{SD}=116 \mu \mathrm{m}, p<0.05)$.

The $\Delta \mathrm{E}$ values of the four materials are shown in Figure 4. The only material to exhibit a clinically noticeable color change was Protemp 3 Garant $(\Delta \mathrm{E}=4.33, \mathrm{SD}=1.19, p$ $<0.001$ ). SNAP (PEMA control; $\Delta \mathrm{E}=1.98, \mathrm{SD}=0.57$ ), Integrity $(\Delta \mathrm{E}=2.61, \mathrm{SD}=0.53)$, and Luxatemp $(\Delta \mathrm{E}=2.89$, $\mathrm{SD}=1.39$ ) all demonstrated $\Delta \mathrm{E}$ values below a clinically perceptible threshold.

\section{Discussion}

The provisional crowns in the present study were constructed using a direct technique. Each crown was fabricated directly over an ivorine abutment tooth and was slightly elevated and depressed in a repeated manner until cured, simulating the direct technique that is used clinically to avoid iatrogenic damage to a vital tooth from the exothermic reaction of the material, as well as to prevent the crown from locking onto the tooth. Our decision to fabricate the provisionals using this technique was based primarily on the belief that this is the method most commonly employed in practice. Potential errors were minimized through the following methods: (1) each crown was fabricated on its own abutment tooth, and (2) a pilot group of temporary crowns was fabricated before the actual experimental crowns to maximize experience in the handling of each type of material. These crowns were not included in the final pool of samples. All provisionals were fabricated over their own respective replica, and were fabricated in a similar environment according to the manufacturer's specifications. Also, all provisionals of the same brand were fabricated on the same day. This was done to achieve standardization of experimental
Figure 2 A tangent was created on each reference point, and lines from the tangent to the margin of the provisional crown and the margin of the prepared ivorine tooth were calculated. The difference between these two measurements was recorded $(\times 10)$.

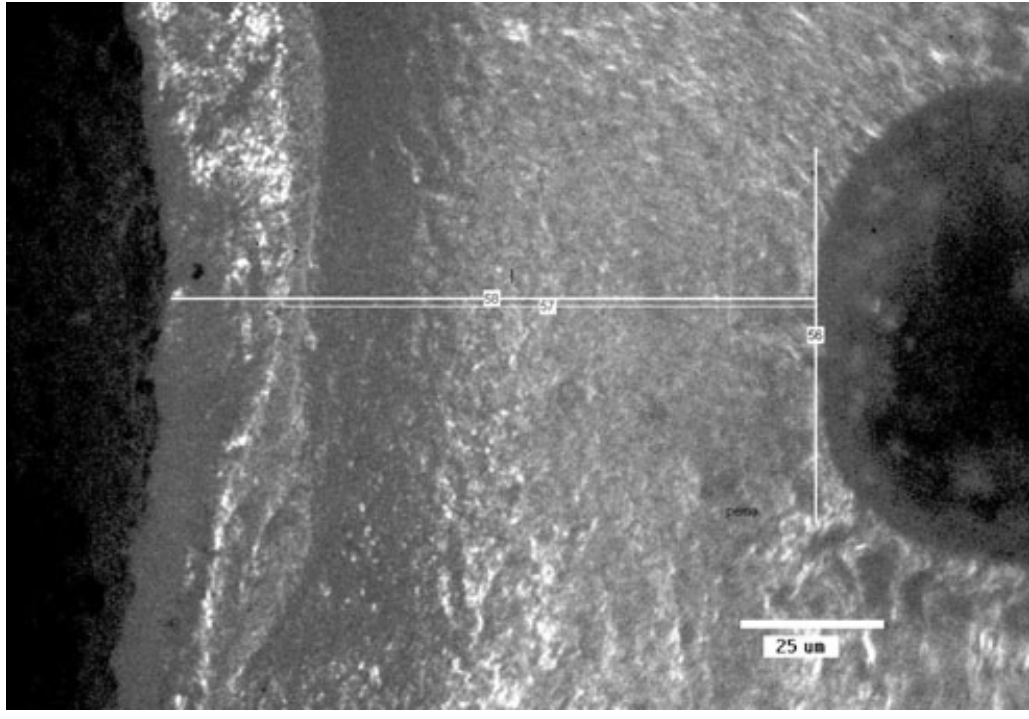




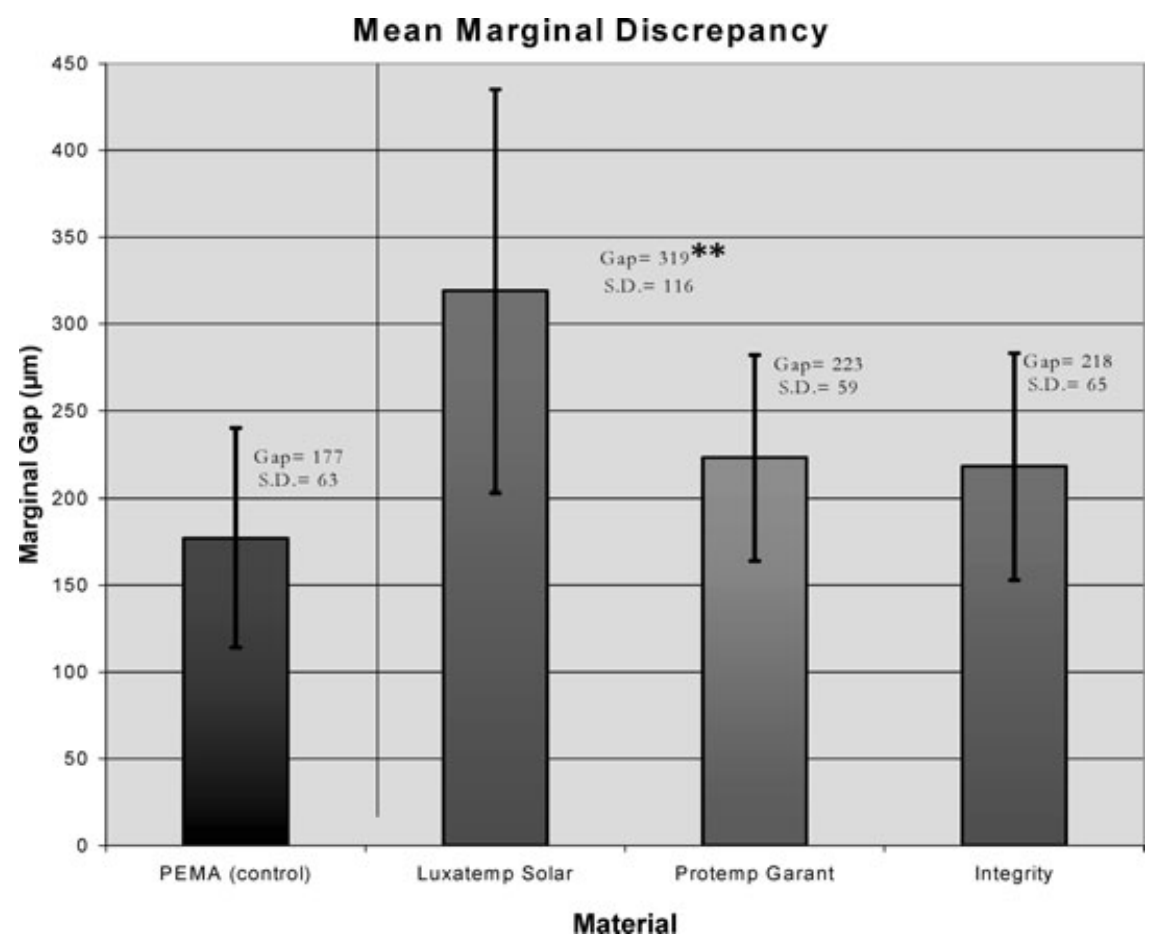

Figure 3 The dual-cured bis-acrylic material, Luxatemp Solar, exhibited significantly greater discrepancy at the margin, compared to the other provisional materials in the study. ${ }^{* *}$ Denotes statistical significance.

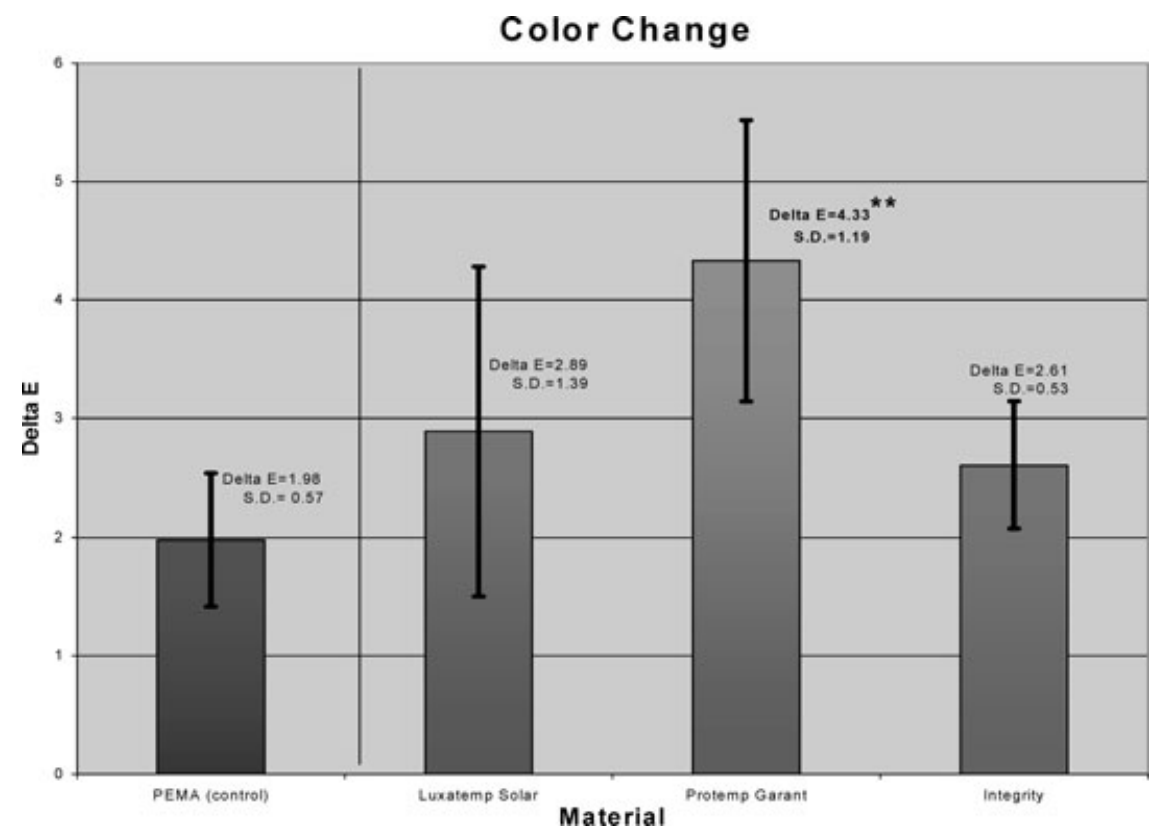

Figure $4 \mathrm{~A} \Delta \mathrm{E}$ value greater than 3.2 is considered a clinically noticeable change in shade of material. The only material in this study to have exceeded this value was Protemp Garant, a bis-acryl composite material. The staining medium used in the study was a dark tea. ${ }^{* *}$ Denotes statistical significance.

conditions. For each provisional crown to be accepted into the final pool of samples, the following inclusion criteria were applied: (1) crowns that did not exhibit internal or external voids; (2) no visible cracks; (3) adaptation to the replica on all surfaces; and (4) of acceptable quality to both evaluators.
As expected, all four materials used in the study exhibited some degree of marginal discrepancy. Luxatemp Solar, the dualcure bis-acryl material, exhibited the greatest discrepancy. Using an indirect technique to fabricate provisional crowns in their study, Koumjian and Holmes found similar results with 
Triad, a dual-cured bis-acryl material. ${ }^{13}$ Results from a study comparing a light-polymerized resin with an auto-polymerizing resin revealed significantly less gap formation with the lightpolymerizing resin. ${ }^{20}$ This might suggest that the majority of gap formation occurs during the auto-cure phase of polymerization of dual-cured materials. Further studies comparing discrepancies between the light-cure and auto-cure phases of dual-cured provisional materials would help assess the level of shrinkage at the margin that may be attributed to each of the phases.

Although the PEMA samples exhibited slightly less discrepancy than samples from both auto-cured bis-acryl groups, this difference was not statistically significant. This finding is contrary to our original hypothesis; however, it is in agreement with a separate study conducted with similar materials. ${ }^{14}$

For the color stability portion of the study, tea was used as the staining medium, as it has previously been found to be one of the strongest staining agents. ${ }^{21}$ Changes in shade were analyzed using the CIE L*a*b* color system. The main advantage of using this system is that it allows a summary value, $\Delta \mathrm{E}$, to be calculated and used as a tolerance for color matching. ${ }^{22}$ In the CIE color system, $\mathrm{L}^{*}$ is defined as the value, or lightness/darkness of the specific color, whereas $a^{*}$ and $b^{*}$ specify the actual hue or color. Positive $\mathrm{a}^{*}$ values indicate a predominance of red hue, while negative values indicate a predominance of green. Positive $b^{*}$ values indicate a tendency towards yellow, whereas values in the negative range demonstrate a bluish tone. With very little exception, most of the samples in each group exhibited positive $a^{*}$ and $b^{*}$ changes in hue, or changes from a green/blue tone to a more red/yellow tone. Positive $a^{*}$ changes for all samples were much lower in magnitude than were positive $b^{*}$ changes. These findings are in agreement with another study that used tea as a staining medium, ${ }^{19}$ and have been attributed to certain red and yellow polyphenolic chromogens. ${ }^{23,24}$

The change in color of our specimens, $\Delta \mathrm{E}$, was calculated from the following formula: $\left[\left(\Delta \mathrm{L}^{*}\right)^{2}+\left(\Delta \mathrm{a}^{*}\right)^{2}+\left(\Delta \mathrm{b}^{*}\right)^{2}\right]^{1 / 2}$, where $\Delta \mathrm{L}^{*}, \Delta \mathrm{a}^{*}$, and $\Delta \mathrm{b}^{*}$ were the differences in the CIE $\mathrm{L}^{*} \mathrm{a}^{*} \mathrm{~b}^{*}$ color parameters between baseline and final color for each sample. Of the four materials, only Protemp Garant, an auto-cured bis-acryl composite, exhibited a clinically noticeable change in shade. This conclusion is based on findings that a $\Delta E$ value greater than 3.2 can be perceived clinically. ${ }^{22}$ The PEMA material was the most resistant to changes in shade. This finding verifies our original hypothesis, and is in agreement with other studies conducted under similar conditions. ${ }^{15,17}$ It contrasts, however, with a study conducted by Sham et al, in which color changes associated with continuous exposure to UV light irradiation were compared. ${ }^{16}$ In their study, the bis-acryl composites demonstrated better resistance to discoloration than the PEMA materials.

\section{Conclusion}

Temporary crowns fabricated from a dual-cure bis-acryl provisional material are likely to exhibit significantly more marginal discrepancy than auto-cure bis-acryl and acrylic resin provisional materials. Dual-cure and some auto-cure bis-acryl pro- visional materials tend to have comparable color stability when compared to a traditional acrylic resin material.

\section{References}

1. Christensen GJ: Provisional restorations for fixed prosthodontics. J Am Dent Assoc 1996;127:249-252

2. Small BW: Pre-treatment wax-ups and provisionals for restorative dentistry. Gen Dent 2005;53:98-100

3. Hernandez EP, Oshida Y, Platt JA, et al: Mechanical properties of four methylmethacrylate-based resins for provisional fixed restorations. Biomed Mater Eng 2004;14:107-122

4. Kaiser DA: Accurate acrylic resin temporary restorations. J Prosthet Dent 1978;39:158-161

5. Diaz-Arnold AM, Dunne JT, Jones AH: Microhardness of provisional fixed prosthodontic materials. J Prosthet Dent 1999;82:525-528

6. Ireland MF, Dixon DL, Breeding LC, et al: In vitro mechanical property comparison of four resins used for fabrication of provisional fixed restorations. J Prosthet Dent 1998;80:158-162

7. Young HM, Smith CT, Morton D: Comparative in vitro evaluation of two provisional restorative materials. J Prosthet Dent 2001;85:129-132

8. Dumbrigue HB: Composite indirect-direct method for fabricating multiple-unit provisional restorations. J Prosthet Dent 2003;89:86-88

9. Miller SD: The anterior fixed provisional restoration: a direct method. J Prosthet Dent 1983;50:516-519

10. Fehling AW, Neitzke C: A direct provisional restoration for decreased occlusal wear and improved marginal integrity: a hybrid technique. J Prosthodont 1994;3:256-260

11. Small BW: Indirect provisional restorations. Gen Dent 1999;47:140-142

12. Bennani V: Fabrication of an indirect-direct provisional fixed partial denture. J Prosthet Dent 2000;84:364-365

13. Koumjian JH, Holmes JB: Marginal accuracy of provisional restorative materials. J Prosthet Dent 1990;63:639-642

14. Tjan AHL, Tjan AH, Grant BE: Marginal accuracy of temporary composite crowns. J Prosthet Dent 1987;58:417-420

15. Yannikakis SA, Zissis AJ, Polyzois GL, et al: Color stability of provisional resin restorative materials. J Prosthet Dent 1998;80:533-539.

16. Sham AS, Chu FC, Chai J, et al: Color stability of provisional prosthodontic materials. J Prosthet Dent 2004;91:447-452

17. Haselton DR, Diaz-Arnold AM, Dawson DV: Color stability of provisional crown and fixed partial denture resins. J Prosthet Dent 2005;93:70-75

18. Doray PG, Wang X, Powers JM, et al: Accelerated aging affects color stability of provisional restorative materials. J Prosthodont 1997;6:183-188

19. Sulieman M, Addy M, Rees JS: Development and evaluation of a method in vitro to study the effectiveness of tooth bleaching. $\mathrm{J}$ Dent 2003;31:415-422

20. Dubois RJ, Kyriakakis P, Weiner S, et al: Effects of occlusal loading and thermocycling on the marginal gaps of lightpolymerized and autopolymerized resin provisional crowns. J Prosthet Dent 1999;82:161-166

21. Khokhar ZA, Razzoog ME, Yaman P: Color stability of restorative resins. Quintessence Int 1991;22:733-737

22. O'Brien WJ: Dental Materials and Their Selection (ed 3). Chicago, IL, Quintessence, 2002, p. 28.

23. Harler CR: Tea Manufacture. London, Oxford United Press, 1963, pp. 13-22.

24. Pearson D: The Chemical Analysis of Foods (ed 7). London, Churchill Livingston, 1976. 